\title{
Is evidence for enhanced mass transfer during dwarf-nova outbursts well substantiated?
}

\author{
Y. Osaki ${ }^{1}$ and F. Meyer ${ }^{2}$ \\ 1 Faculty of Education, Nagasaki University, Nagasaki 852-8521, Japan \\ 2 Max-Planck-Institut für Astrophysik, Karl Schwarzschild Str. 1, 85740 Garching, Germany
}

Received 16 October 2002 / Accepted 21 January 2003

\begin{abstract}
Outburst mechanisms of SU UMa-type dwarf novae are discussed. Two competing models were proposed; a pure disk instability model called the thermal-tidal instability model (TTI model) and the enhanced mass transfer model (EMT model). Observational evidence for enhanced mass transfer from the secondary star during outbursts is critically examined. It is demonstrated that most evidence for enhanced mass transfer is not well substantiated. Patterson et al. (2002) have recently claimed to have found evidence for enhanced mass transfer during the 2001 outburst of WZ Sge. We show that their evidence is probably due to a misinterpretation of their observed light curves. Our theoretical analysis also shows that irradiation during outburst should not affect the mass transfer rate. A refinement of the TTI model is proposed that can explain why superhumps appear a few days after the superoutburst maximum in some SU UMa stars. We present our own interpretation of the overall development of the 2001 outburst of WZ Sge based on the TTI model that does not require the assumption of an unproved enhanced mass transfer.
\end{abstract}

Key words. accretion, accretion disks - binaries: close - novae, cataclysmic variables - stars: dwarf novae stars: individual: WZ Sge

\section{Introduction}

SU UMa stars are dwarf novae of short orbital periods that show two distinct types of outbursts, called short normal outburst and long superoutburst (see, e.g., Warner 1995 for dwarf novae in general and SU UMa stars in particular). In ordinary SU UMa stars several short normal outbursts are sandwiched between two consecutive superoutbursts. The cycle from one superoutburst to the next is called supercycle. During the superoutburst periodic humps called superhumps appear that repeat with a period slightly longer than the binary orbital period. The superhump phenomenon in SU UMa stars is now well understood in terms of the tidal instability (Whitehurst 1988; Hirose \& Osaki 1990; Lubow 1991): superhumps are produced by periodic tidal stressing of the eccentric precessing accretion disk which is formed by the 3:1 resonance tidal instability.

The short normal outburst of SU UMa stars is thought to be essentially the same as the outburst of U Gem stars and is believed to be caused by the thermal instability in the accretion disk. For the superoutburst and supercycle of SU UMa stars Osaki (1989) has proposed the thermal-tidal instability model (TTI model) in which the ordinary thermal instability is coupled with the tidal instability. Based on the TTI model, Osaki (1996) has presented a unification model for outbursts of dwarf novae in which a rich variety of outburst behaviors of

Send offprint requests to: Y. Osaki,

e-mail: osaki@net.nagasaki-u.ac.jp non-magnetic cataclysmic variable stars is understood within the general framework of the disk instability model.

Although the TTI model is basically successful in explaining the superoutburst phenomenon of SU UMa stars, there still remains some deficiency. Hellier (2001) has suggested a minor modification to the TTI model in the case of binary systems with extremely low mass ratio. Moreover, objections to the TTI model were raised by $\operatorname{Smak}(1996,2000)$ and by Lasota's group (see Lasota 2001 for a review of his group's view of this problem). They argue that enhanced mass transfer, likely caused by irradiation of the secondary star by the central source of radiation, is responsible for the superoutburst of SU UMa stars (EMT model). Historically speaking, an enhanced mass transfer model by an irradiated secondary was proposed by Osaki (1985) to explain superoutbursts of SU UMa stars but this model was later abandoned by its author in favor of the TTI model (Osaki 1996). On page 486 of his (2001) review, Lasota made the following statement: "the main deficiency of the TTI model is that it neglects a property of SU UMa stars and dwarf novae outbursts in general: the observed enhancement of the mass-transfer rate during outbursts."

The purpose of this paper is to examine critically whether Lasota's statement, i.e., the enhancement of the mass-transfer during outbursts, is observationally and theoretically substantiated. Patterson et al. (2002) recently presented extensive photometry observations of the 2001 superoutburst of WZ Sge. 
From eclipse observations during the main outburst of WZ Sge in 2001 they claim that the mass transfer rate was enhanced by a factor of up to 60 from its quiescent value. We also discuss these observations and their interpretation.

In Sect. 2, we examine the various claims for enhanced mass transfer and we show that most evidence is not well founded. Our theoretical analysis in Sect. 3 shows severe problems for any model that claims an effect of irradiation on the mass transfer rate in outburst. In response to Smak's (1996, 2001) criticism of the TTI model, we propose in Sect. 4 a refinement of the TTI model that explains why superhumps appear a few days after the superoutburst maximum in some SU UMa stars. After thus having demonstrated that enhanced mass transfer during outburst is not well substantiated, we present our own interpretation for the overall development of the 2001 outburst of WZ Sge in Sect. 5. In the Appendix, we critically discuss the new interpretation of the early hump phenomenon by Kato (2002). A conclusion of the paper is given in Sect. 6.

\section{Examination of claimed observational evidence for enhanced mass transfer during outburst}

\subsection{Early hump phenomenon in WZ Sge}

Periodic humps appeared during the first 10 days of the 1978 outburst of WZ Sge that repeated with the orbital period. These humps have been called either "outburst orbital humps" or "early superhumps". Here we call them simply "early humps" (see the discussion in Osaki \& Meyer 2002). It is interesting to note that the early hump phenomenon was used as evidence for enhanced mass transfer in two different ways by Patterson et al. (1981) and by Smak (1993).

Patterson et al. (1981) interpreted the humps repeating with the orbital period in the first 10 days of the 1978 outburst as brightening of the hot spot caused by enhanced mass transfer. However they had a difficulty with this interpretation since the position of the hot spot would have had to move azimuthally by 60 degrees from its quiescent position. The double hump nature of early humps observed in the 2001 outburst of WZ Sge also is difficult to explain by a hot spot brightening (Osaki \& Meyer 2002).

Smak (1993) on the other hand took the early humps observed in the $1978 \mathrm{WZ}$ Sge outburst as evidence for a heated secondary. But also he had a difficulty with the phase of maximum visibility of the irradiated surface of the secondary star. Nevertheless, he calculated the temperature of the heated surface of the secondary star by assuming that radiation of the central source intercepted by the secondary star is thermalized in the photosphere and raises the surface temperature of the secondary star. This point will be discussed in the next section.

However, if the early hump phenomenon is of disk origin due to a non-axisymmetric structure produced by tidal effects in our 2:1 resonance model (Osaki \& Meyer 2002) or in Kato's model (Kato 2002), the evidence for a brightened hot spot or for a heated secondary star disappears. Thus the early hump phenomenon of WZ Sge stars can not be taken as evidence for enhanced mass transfer from the secondary star.

\subsection{Enhanced humps in normal outbursts presented by Vogt (1983)}

One piece of evidence for enhanced mass transfer referred to by Smak (1996) was found in the observational review of VW Hyi by Vogt (1983). Vogt noted that significant enhancements in the hump amplitude during short eruptions were present in only four of nine cases and that all four cases were confined to those occurring less than 40 days from the next superoutbursts, summarized in Fig. 4 of his paper.

In Vogt's picture, enhanced humps observed in maximum or decline of normal short eruptions of VW Hyi were due to brightening of the hot spot and the enhanced amplitudes of the hot spot were evidence for an enhanced mass transfer from the secondary star. We question Vogt's interpretation of the enhanced orbital humps in these four cases. Instead we suggest that the observed humps rather are of superhump nature in an embryonic form. By superhumps in an embryonic form we mean superhumps in the early stage of their growth. Such embryonic superhumps were observed in precursor outbursts in T Leo by Kato (1997) and in V436 Cen by Semeniuk (1980).

Observations discussed below were done a quarter of a century ago and this question can be easily settled by new observations with CCD. We urge observers to make such observations.

In fact, the normal outburst, claimed by Vogt (1983) to have exhibited a 15 times increase in hot spot brightness, was the precursor outburst of the May 1977 superoutburst of VW Hyi observed by Marino \& Walker (1979). If we compare the light curve of this superoutburst (Fig. 4 of Marino \& Walker 1979) with those of the 1993 January superoutburst of T Leo observed by T. Kato (Figs. 1 and 2 of Kato 1997), we find they are very similar, exhibiting basically the same phenomenon. Kato (1997) has found that the hump period during decline from the precursor outburst of T Leo in January 1993 is 2.4\% longer than the orbital period and thus these humps are embryonic superhumps rather than orbital humps. In the case of the precursor outburst in the decline stage of the May 12, 1977 outburst observed by Marino and Walker, only three hump maxima were seen and it would be difficult to determine their exact period in order to decide whether they are enhanced orbital humps or embryonic superhumps.

Marino \& Walker (1979) found another hump-like feature in the decline of the 1978 October normal outburst of VW Hyi which occurred 160 days after the previous superoutburst (the mean supercycle of VW Hyi is 180 days), but they were puzzled because the observed hump feature looked like a superhump with a period similar to that of superhumps rather than that of orbital humps. We interpret these humps as indeed embryonic superhumps that failed to develop into fully-grown superhumps and superoutburst. Two of the four cases claimed to exhibit an enhanced hot spot by Vogt (1983) were the same two observations by Marino \& Walker (1979) discussed above.

The other two cases were those observed by Haefner et al. (1979). These two are outbursts designated as No. 280 and No. 281 which occurred around Nov. 15 and Nov. 26, 1974 while the next superoutburst designated as No. 282 occurred around Dec. $7 / 8$ in 1974. With respect to the outburst No. 281, light curves for three consecutive nights were shown in Fig. 4 
of Haefner et al. (1979). The phases of the hump maxima in the first and third night agreed with those expected from the quiescent hump ephemeris while the phase of the biggest hump in the second night was shifted by 0.5 from that expected. Haefner et al. (1979) interpreted this hump maximum observed in the second night as a secondary maximum sometimes seen in quiescent orbital humps. However, another interpretation is possible. If we combine the hump maxima in all three nights in one ephemeris, we would get a hump period exceeding the orbital period by $3.8 \%$ (i.e., a beat period of 2 days between the orbital and superhump periods). Thus these humps in outburst No. 281 of VW Hyi could well be interpreted as embryonic superhumps which failed to develop into fully-grown superhumps rather than as orbital humps. No light curves for the outburst No. 280 of VW Hyi are available in published form. However, the enhancement of hump amplitude in this outburst claimed by Vogt (1983) was rather small (the smallest among the four cases, i.e., enhancement less than 5 in Vogt 1983) and we do not discuss this outburst any further.

In conclusion, the evidence for enhanced mass transfer presented by Vogt (1983) is not sufficient to substantiate the claim that the mass transfer rate is enhanced in the maximum and decline stages of normal outbursts if they occur near the next superoutburst.

\subsection{Eclipse observations of the 2001 superoutburst of WZ Sagittae and Patterson's et al. (2002) interpretation for enhanced mass transfer}

Patterson et al. (2002) have presented extensive photometric observations of the 2001 outburst of WZ Sge. During a first stage of about 12 days early humps (outburst orbital humps or "OOH" in Patterson et al. 2001) were visible. This stage is discussed in Osaki \& Meyer (2002). In the following stage common superhumps were observed. Eclipses suddenly appeared with the growth of the superhumps and were visible all the way from the main outburst stage through the so called echo outburst stage up to the final decline.

By synchronous summation of the superhump light curves at the orbital period through out the beat phase between orbital and superhump period Patterson et al. (2002) obtained "orbital lightcurves" that looked like those of the quiescent orbital hump (lowest right panel of their Fig. 7 and left panel of their Fig. 16). Therefore the authors interpreted them as hot spot light curves and the eclipses observed as eclipses of the mass transfer hot spot. By comparing intensity amplitudes of the hump light they concluded that the mass transfer rate during this stage had increased by a factor up to 60 above the quiescent level. This would strongly challenge the TTI model of SU UMa star outbursts.

We now give evidence that the orbital hump simply results from the orbital aspect of the superhump dissipation pattern in high inclination systems like WZ Sge, and that the observed eclipses are eclipses of the superhump light source itself. Thus an increased hot spot brightness and the inferred increased mass transfer rate are spurious, the result of a misinterpretation of observed light curve.
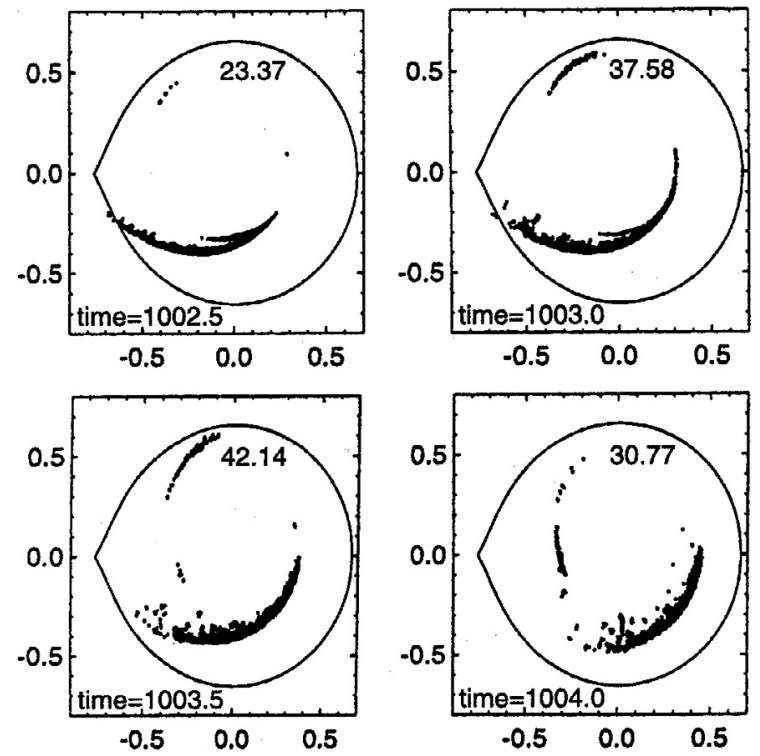

Fig. 1. Superhump dissipation pattern (black dots) in hydrodynamic simulations for four different superhump phases around superhump maximum. Axes: Cartesian coordinates in orbital plane in units of binary separation. Contour line: primary Roche lobe; time: time elapsed in simulation in units of orbital period $/ 2 \pi$; number within Roche contour: strength of dissipation (arbitrary unit). After Murray (1999).

We first note that the basic pattern of light curves during the common superhump stage repeats more or less with the superhump beat period of roughly 5 days. Eclipses were visible for only half of these beat phases. Eclipse characteristics such as eclipse depth, eclipse width, and time of mid-eclipse in the orbital light curve all were functions of the beat phase (see Fig. 21 of Patterson et al. 2002).

Now the superhump light variation is an intrinsic variation produced by time varying dissipation as the secondary orbits the eccentric disk. But the dissipation pattern is also extremely non-axisymmetric as can be seen e.g. in hydrodynamic simulations by Hirose \& Osaki (1990, their Fig. 7b) and Murray (1998, his Fig. 8). Figure 1 shows the superhump dissipation pattern for the four (out of nine) phases around superhump maximum from Murray's Fig. 8. The most conspicuous feature in the simulations is the appearance of an arch-like tail that extends near to the edge of the primary Roche lobe when the dissipation is strong (near superhump maximum). Part of this arch-like tail can be eclipsed in the case of WZ Sge. When the tidal dissipation is weak (superhump minimum) no conspicuous feature in the tidal dissipation is visible and as far as the dissipative light is concerned the disk looks compact and circular.

The tidal dissipation of the superhumps occurs near the disk rim in a region about $1 / 10$ of the disk radius wide (see Fig. 1). This is the same size as the estimated half thickness of the disk at its rim. Thus about one half of the superhump light may be expected to be radiated from the two horizontal disk surfaces and one half from a more or less vertical disk rim. The radiation pattern of the latter part is strongly dependent on the orbital aspect of the observer. 


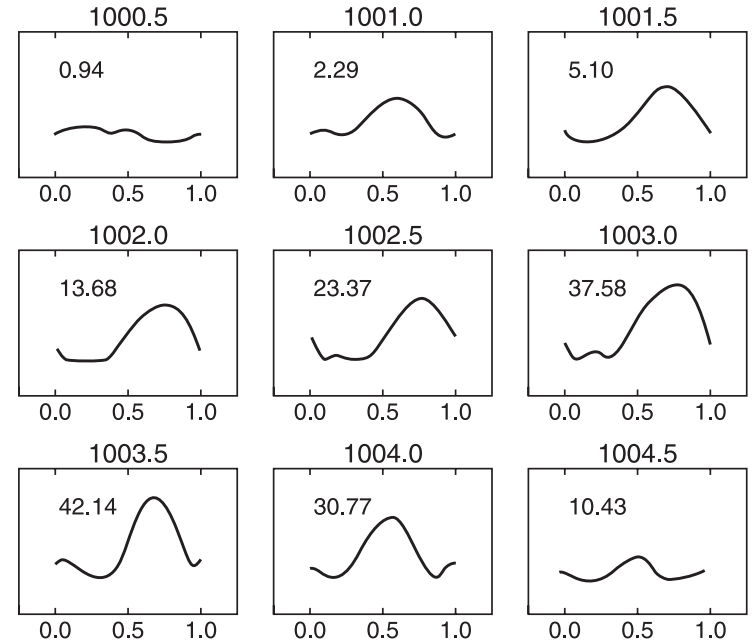

Fig. 2. Estimated orbital light curves from superhump dissipation pattern projected for an observer at high inclination for 9 different superhump phases from Murray's (1999) simulation. Horizontal axis: orbital phase; vertical axis: intensity in arbitrary units; number on top: time elapsed in simulation in units of orbital period $/ 2 \pi$; number inside frame: strength of dissipation (arbitrary unit).

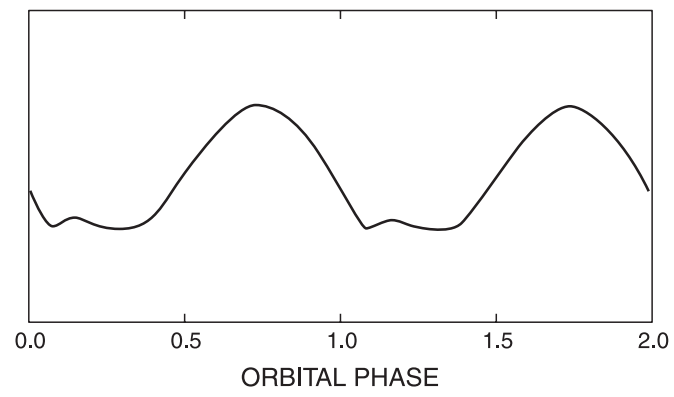

Fig. 3. Superposition of the nine light curves of Fig. 2 weighted by their relative dissipation strength. Horizontal axis: orbital phase; vertical axis: intensity (arbitrary unit). This corresponds to a synchronous summation at the orbital period of observed superhump light curves.

We have estimated how much light an observer near the orbital plane would receive from this component due to the projection of its radiating surface at the various orbital phases for all nine superhumps phases displayed in Fig. 8 of Murray (1998). The resulting orbital light curves are shown in Fig. 2. Note the strong variation of the total dissipated light given by the numbers inside the individual graphs. An intensity weighted superposition of these graphs yields an "orbital light curve", that corresponds to the synchronous superposition of the superhump light curves at the orbital period by Patterson et al. Figure 3 gives the resultant light curve. It shows a hump with maximum phase around 0.75 and extending over half the orbital periods in fairly good agreement with the observed light curve shown in Fig. 7 of Patterson et al. (2002). The strong asymmetry of the superhump dissipation pattern thus naturally can explain the apparent orbital hump and no increased hot spot light is needed.

There is even intrinsic evidence in Patterson's et al. (2002) data that the observed orbital light curves and eclipses are indeed due to the superhump light source and not due to a mass transfer hot spot. In Fig. 18 Patterson et al. (2002) show the changes of eclipse depth and orbital wave height as evidence for a changing mass transfer rate. However these two variations closely follow the variation of the superhump light amplitude itself, listed in their Table 3. This indicates that it is the superhump light source which is eclipsed and whose aspect varies with the orbital phase rather than a hot spot.

Why are eclipses not observed in the first 12 days of the outburst when the early humps (or "OOH") were seen? Osaki $\&$ Meyer (2002) have suggested that early humps are produced by the two-armed dissipation pattern of the 2:1 Lindblad resonance. Though at the disk rim this spiral dissipation pattern is located azimuthally far away from the secondary star (Fig. 3a in Lin \& Papaloizou 1979). Thus it is fairly likely that this light is not eclipsed.

We conclude that there is no evidence for enhanced mass transfer in the common superhump stage of the 2001 outburst of WZ Sge.

Another case quoted by Smak (1996) as evidence for enhanced mass transfer are eclipse observations during the superoutburst of Z Cha by Warner \& O'Donoghue (1988). O'Donoghue (1990) analyzed these observations and found that, with the exception of one case, the superhump light source was not confined to the hot spot region. In one exceptional case called eclipse 77878 (Fig. 2a in Warner \& O'Donoghue 1988), he found that all superhump light was confined to a region near the position of the quiescent hot spot. O'Donoghue (1992) concluded that "as the bright spot during this eclipse is more than twenty times as luminous as at quiescence, a brief period (due to the rarity of this kind of eclipse) of enhanced mass transfer is the most natural explanation".

However, if we recall that the superhump light source is extremely non-axisymmetric and mostly confined to an arch-like tidal tail extending in the following lune of the accretion disk, it is most natural to assume that the light source eclipsed in eclipse 77878 was just the superhump light source itself rather than the hot spot.

In fact, as the light curve of eclipse 77878 in Warner \& O'Donoghue (1988) shows, the eclipse occurred at the rising phase to the superhump maximum and the shoulder of the superhump light curve was eclipsed. We may conclude that what was eclipsed in this case is also the superhump light source itself and not a hot spot, and that there is no evidence for enhanced mass transfer in this case either.

\section{Theoretical aspects for enhanced mass transfer from irradiated secondary stars}

In the preceding sections we have shown that all claimed evidence for increased mass transfer in outbursts of cataclysmic variables is inconclusive, partially contradictory, and in all cases can be explained otherwise, in fact supporting the TTI model in its various features.

What is the situation from the theoretical side? The luminosity often rises in outburst by orders of magnitude and one might expect strong irradiation of the secondary star, raising the surface temperature and lifting gas higher up in the gravitational potential. Why should this not lead to a density 
increase in the Lagrange point $\mathrm{L}_{1}$ and an increase of the mass overflow rate? There are however well known difficulties with this simple picture, and we show in the following that simple quantitative estimates in the complex physical situation yield prohibitive limits on the increase of the mass overflow. We will perform the estimates for the case of WZ Sge. This should be a particularly sensitive system for such effects because of the possibly very low surface temperature of its secondary star and an extremely high luminosity increase in the outburst.

The main problems with an irradiation effect on the mass transfer are due to (1) the shadow that the accretion disk casts on the $L_{1}$ point and (2) the high opacity in the extreme ultraviolet band which prevents the bulk of the radiative flux from reaching the stellar photosphere at all. In addition (3) the light that penetrates to the stellar surface heats up the subsurface layers but this heated material cannot effectively move towards the shadowed $\mathrm{L}_{1}$ point since strong Coriolis forces prevent it from moving into the lower pressure region there.

\subsection{The shadow of the accretion disk}

Light sources are the white dwarf, the inner accretion disk and possibly a corona above the inner disk. Important for our analysis of the effect of irradiation is to which degree the disk casts a shadow on the region around $\mathrm{L}_{1}$.

Disk structure computations allow determination of the thickness of the accretion disk in the outburst. Near the outer radius $r_{\mathrm{d}}$ of the disk at $10^{10.4} \mathrm{~cm}$ around a white dwarf of mass $M_{\mathrm{WD}}=0.7 M_{\odot}$ and for an estimated accretion rate for WZ Sge in the early phase of outburst of $10^{-7} M_{\odot} / \mathrm{yr}$ one obtains (Meyer \& Meyer-Hofmeister 1982) for the height of the disk photosphere above the midplane

$H_{\mathrm{d}}=10^{9.4} \mathrm{~cm}$.

The height varies weakly with the accretion rate, proportional to $\dot{M}^{1 / 6}$. This height $H_{\mathrm{d}}$ is larger than the radius of the white dwarf

$R_{\mathrm{WD}}=10^{8.9} \mathrm{~cm}$

(Nauenberg 1972). It is also larger than the vertical extent of the mass transfer cross section in the $\mathrm{L}_{1}$ point. The latter is obtained as the vertical scaleheight of a gas column of temperature $T_{0}$ in the Roche geometry at $\mathrm{L}_{1}$ (e.g. Meyer \& Meyer-Hofmeister 1983; Kolb \& Ritter 1990). For the assumed mass ratio $q=0.1$ and a surface temperature $T_{0}=10^{3.2} \mathrm{~K}$ of the secondary this gives

$H_{\mathrm{L}_{1}}=10^{8.3} \mathrm{~cm}$.

The value varies proportional to $T_{0}^{1 / 2}$. This means the region around $\mathrm{L}_{1}$ is in the shadow of the disk rim. Even light from a corona above the inner disk is shielded by the disk rim since the height $H_{\mathrm{d}}$ of the accretion disk rim is also larger than the scaleheight of a corona $H_{\mathrm{c}}$ above the inner accretion disk, for a coronal temperature of $10^{8} \mathrm{~K}$ at the distance $r=10^{9} \mathrm{~cm}$ from the white dwarf

$H_{\mathrm{c}}=\left(\frac{2 \frac{\mathfrak{R}}{\mu} \operatorname{Tr}^{3}}{G M}\right)^{1 / 2}=10^{8.7} \mathrm{~cm}$ with $\mathfrak{R}$ gas constant, $\mu$ molecular weight, $G$ gravitational constant.

We conclude that the region of mass transfer at $\mathrm{L}_{1}$ lies deep in the shadow band that extends $10^{9.4} \mathrm{~cm}$ to both sides of the equator of the secondary star for any light that comes from the white dwarf, an inner disk or a corona above the inner disk.

\subsection{The irradiated part of the surface of the secondary star}

While the mass transfer point $\mathrm{L}_{1}$ lies in the center of the broad shadow of the accretion disk and receives no direct light the possibility remains that irradiation raises the temperature outside of this shadow zone and that by exchange of heated material from the irradiated part with cool material in the shadow zone below $\mathrm{L}_{1}$ the temperature also rises there. That could still result in an increased mass transfer. We now discuss the physical aspect of this situation in order to see whether there is an effect on the mass transfer rate.

First we derive an estimate for the temperature of the irradiated surface of the secondary star. In quiescence the surface receives the radiation from the hot white dwarf, $T_{\mathrm{WD}}=15000 \mathrm{~K}$ (Cheng et al. 1997). If this radiation is fully absorbed and reradiated from the secondary surface as black body radiation its temperature at the distance closest to the white dwarf would be

$T_{\text {irr }}=\left(\frac{R_{\mathrm{WD}}}{a x_{\mathrm{L}}}\right)^{1 / 2} \cdot T_{\mathrm{WD}}=10^{3.4} \mathrm{~K}$

where $x_{\mathrm{L}}=r_{\mathrm{L}} / a=0.72$ is the distance of $\mathrm{L}_{1}$ from the primary in units of the separation $a$, here for the assumed mass ratio $q=0.1$.

In outburst, with the accretion rate $\dot{M}=10^{-7} M_{\odot} / \mathrm{yr}$, the accretion luminosity of the white dwarf will rise to the value

$L_{\mathrm{WD}, \text { outburst }}=\frac{1}{2} \frac{G M_{\mathrm{WD}} \dot{M}}{R_{\mathrm{WD}}}=10^{35.6} \mathrm{erg} / \mathrm{s}$.

The corresponding effective temperature is

$T_{\text {WD,outburst }}=\left(\frac{L_{\mathrm{WD}}}{4 \pi \sigma R_{\mathrm{WD}}^{2}}\right)^{1 / 4}=170000 \mathrm{~K}$.

Radiation of this temperature lies in the extreme ultraviolet and supersoft X-ray range with wavelengths around $200 \AA$ where opacities are very large. Such radiation cannot reach the surface of the secondary star but is absorbed and reradiated in high layers of the secondary's atmosphere (see Suleimanov et al. 1999 for the analogous case of supersoft X-ray sources for a detailed discussion). Hard X-ray flux above $2 \mathrm{keV}$ on the other hand could penetrate to the photosphere. But RXTE observations between 2 and $6 \mathrm{keV}$ yielded upper limits of $f_{\mathrm{x}}=5.3 \times 10^{-12}$ and $3.7 \times 10^{-12} \mathrm{erg} / \mathrm{cm}^{2} \mathrm{~s}$ (for the flux density at earth) on the day of discovery and 4 days into the last outburst of WZ Sge (Kuulkers et al. 2002). With a distance to WZ Sge of about $d=$ 50 pc (Smak 1993; Meyer-Hofmeister et al. 1998) this corresponds to upper limits for the X-ray luminosity

$L_{\mathrm{x}}=4 \pi d^{2} f_{\mathrm{x}} \leq 10^{30.2} \mathrm{erg} / \mathrm{s}$, 
an order of magnitude less than even the luminosity of $10^{31.4} \mathrm{erg} / \mathrm{s}$ of the $15000 \mathrm{~K}$ quiescent white dwarf. Contrary to the extreme UV, near UV and optical radiation from the accretion disk and white dwarf however can reach the photosphere of the secondary and raise its temperature. From HST observations of WZ Sge (Kuulkers et al. 2002) 8 days into the 2001 outburst we estimate a flux density longward of $\lambda=$ $2000 \AA$ of $f_{\mathrm{UV}^{\prime}}=10^{-8.5} \mathrm{erg} / \mathrm{cm}^{2} \mathrm{~s}$ at earth. For a distance of $50 \mathrm{pc}$ this corresponds to a luminosity

$L_{\mathrm{UV}^{\prime}}=4 \pi d^{2} f_{\mathrm{UV}}{ }^{\prime}=10^{33.0} \mathrm{erg} / \mathrm{s}$.

If this radiation is reprocessed at the secondary's photosphere it raises the surface temperature to

$T_{\text {irr }}=\left(\frac{L_{\mathrm{UV}^{\prime}}}{4 \pi\left(a x_{\mathrm{L}}\right)^{2} \sigma}\right)^{1 / 4}=10^{3.8} \mathrm{~K}$

at the distance $a x_{\mathrm{L}}$ of $\mathrm{L}_{1}$ from the white dwarf. This is a maximal value. Surface elements farther away from the central source and inclined to the direction of irradiation will have a lower surface temperature.

It is worth noting that this temperature estimate of $10^{3.8} \mathrm{~K}$ is significantly lower than the value of $10^{4.32} \mathrm{~K}$ that Smak (1993) derived from spectral fits to IUE observations early in the December 1978 outburst of WZ Sge. He attributed this component to the irradiated surface of the secondary star but noted an unexplained inconsistency between the observed and expected phases of this component in the light curve.

Our significantly lower estimate for the surface temperature of the secondary star in outburst provides additional support that the source of the component determined by Smak is not the surface of the irradiated secondary but is the $2: 1$ resonance dissipation pattern at the rim of the accretion disk as suggested in Osaki \& Meyer (2002). For that dissipation pattern a temperature around $10^{4.3} \mathrm{~K}$ was estimated. The heat deposited by irradiation at the surface of the secondary spreads with time by radiative diffusion into the subsurface layers. The depth down to which the temperature is raised depends on the opacity of the gas and the duration $t$ of the irradiation. Using standard radiative diffusion and opacities in the temperature range between $10^{3.2} \mathrm{~K}$ and $10^{3.8} \mathrm{~K}$ taken from Alexander et al. (1983) we estimate the column density of the affected layer as

$\Sigma=10^{2.8}\left(\frac{t}{3^{\mathrm{d}}}\right)^{1 / 2} \mathrm{~g} / \mathrm{cm}^{2}$

This is smaller than the column density $\Sigma=10^{3.9} \mathrm{~g} / \mathrm{cm}^{2}$ down to the level where the unirradiated star of surface temperature $10^{3.2} \mathrm{~K}$ itself reaches the $10^{3.8} \mathrm{~K}$ of the irradiated surface. For this estimate we used a value of $\mathrm{d} \ln T / \mathrm{d} \ln P=0.15$, a mean value near the surface of very cool low mass stars (Búning, private communication) and a pressure at the surface $P_{\mathrm{s}}$ of $10^{3.7} \mathrm{dyn} / \mathrm{cm}^{2}$ (which for WZ Sge corresponds to a mass overflow rate of $10^{-11} M_{\odot} / \mathrm{yr}$ ).

Thus below the irradiated surface of the secondary a more or less isothermal layer of $T=10^{3.8} \mathrm{~K}$ is formed.

\subsection{Heat transport from irradiated to shadowed regions?}

The heating of the subsurface material under the irradiated surface raises the pressure and causes pressure differences on equipotential surfaces between irradiated and unirradiated parts. They tend to drive heated material into the shadow belt and below $L_{1}$ which could raise the pressure there and lead to an increase of the mass transfer rate.

However, the system is rotating with the binary period and due to deformation of the stellar surface near $\mathrm{L}_{1}$ the rotation frequency has a component normal to the surface. For $q=$ 0.1 along the meridian through $\mathrm{L}_{1}$ one obtains for this normal component

$\Omega_{\perp}=\Omega_{\mathrm{orb}} \cdot \cos \vartheta=0.56 \Omega_{\mathrm{orb}}$

where $\Omega_{\text {orb }}=2 \pi / P_{\text {orb }}=10^{-2.9} \mathrm{~s}^{-1}$ is the orbital angular frequency ( $P_{\text {orb }}$ orbital period) and $\vartheta$ is the angle between the surface normal and the rotation axis of the system. In the Roche geometry this angle changes gradually as one moves away from the meridian. But along the meridian is the shortest path out of the shadow band and the driving pressure gradient is the largest in this direction. We will show now that rotation practically prevents heated matter to flow into the low pressure region below $\mathrm{L}_{1}$. For this we simplify the curved shape of the equipotential surfaces near $L_{1}$ by adopting a locally plane geometry with Euclidian coordinates $x$ in the direction of constant latitude, $y$ in the direction of constant longitude, and $z$ in the direction of the outward surface normal.

The horizontal equations of motion in this system are used by Spruit (2002) in the context of a solar problem. There it is shown that the equations can be simplified if the vertical extent is small compared to the latitudinal extent which is the case here with a few vertical pressure scaleheights of order $10^{8} \mathrm{~cm}$, small compared to the width of the shadow band of $10^{9.4} \mathrm{~cm}$. Since the main gradients are perpendicular to the shadow band which spreads along the equator we neglect gradients in the $x$ direction and obtain (Pedlosky 1982; Spruit 2002)

$$
\begin{aligned}
-\frac{\partial P}{\partial y}-2 \rho v_{x} \Omega_{\perp}+\frac{\partial}{\partial z}\left(\rho v \frac{\partial v_{y}}{\partial z}\right) & =0, \\
2 \rho v_{y} \Omega_{\perp}+\frac{\partial}{\partial z}\left(\rho v \frac{\partial v_{x}}{\partial z}\right) & =0 .
\end{aligned}
$$

$v$ is the kinematic viscosity. Without viscosity we have the "geostrophic" flow along the shadow band

$v_{x}=-\frac{1}{2 \Omega_{\perp}} \frac{1}{\rho} \frac{\partial P}{\partial y} \approx-\frac{1}{2 \Omega_{\perp}} \frac{\Delta V_{\mathrm{s}}^{2}}{l}$
$v_{y}=0$

where $V_{\mathrm{s}}=(P / \rho)^{1 / 2}$ is the isothermal sound velocity and $l$ is the half width of the shadow band. Thus the flow velocity is at right angle to the driving pressure gradient which is balanced by the Coriolis force, similar to flows in weather systems and oceans on the rotating earth. Thus without friction no heat can be advected into the shadow region below $\mathrm{L}_{1}$.

If a small viscosity is present the geostrophic flow is accompanied by a small drift into the low pressure shadow 
band. Replacing $z$ derivatives by division by the vertical scale height $H$ in the equation for $v_{y}$ one obtains

$v_{y}=\frac{v}{2 \Omega_{\perp} H^{2}} v_{x}=-\frac{v \Delta V_{\mathrm{s}}^{2}}{4 \Omega_{\perp}^{2} H^{2} l}$.

What is the value of a possible viscosity? Molecular viscosity is negligibly small. Can there be turbulent viscosity? The layer irradiated and heated from above is stable against convection as the temperature is constant or even increasing upwards. This suggests that there will be practically no viscosity in the main body of the heated material. Only at the bottom (i.e. its densest layer) one might have to consider that convection from the original stellar envelope below penetrates into the heated layer above. We will now estimate the effect that this will have on the advection of heat into the shadow zone. For this we determine from mixing length theory a "convective" viscosity

$v=\frac{1}{3} l_{\mathrm{m}} v$

where $l_{\mathrm{m}}$ is the mixing length, e.g. the pressure scaleheight $H$, and $v$ is the convective velocity of the cool secondary at the depth where the overlying irradiation heated zone starts. The velocity $v$ follows from the requirement that $v$ transports the convective flux, which is equal to the flux $\sigma T_{0}{ }^{4}$ radiated away at the surface of the unirradiated secondary. Using the value of $\mathrm{d} \ln T / \mathrm{d} \ln P=0.15$ to determine the specific heat and for a density $\rho=\Sigma / H=10^{-4.2} \mathrm{~g} / \mathrm{cm}^{3}\left(\Sigma=10^{2.8} \mathrm{~g} / \mathrm{cm}^{3}\right.$, Eq. (11), and $H=10^{7} \mathrm{~cm}$ ) one obtains (Kippenhahn \& Weigert 1990, Eqs. (7.6) and (7.7)) $v=10^{3.5} \mathrm{~cm} / \mathrm{s}$. The value for $H$ results from the surface gravity in Roche geometry at the distance $l=10^{9.4} \mathrm{~cm}$ of the shadow band boundary from the Lagrange point $L_{1}$ and for a temperature of $10^{3.8} \mathrm{~K}$. Equation (16) then gives

$v=10^{10} \mathrm{~cm}^{2} / \mathrm{s}$.

The pressure ratio between surface, $P_{\mathrm{s}}=10^{3.7} \mathrm{dyn} / \mathrm{cm}^{2}$, and the bottom layer, $P=\frac{\mathfrak{R}}{\mu} T \rho=10^{7.4} \mathrm{dyn} / \mathrm{cm}^{2}$, determines the temperature ratio between surface and bottom and with the surface temperature of the unirradiated star $T_{0}=10^{3.2} \mathrm{~K}$ this gives $T=T_{0}\left(P / P_{\mathrm{s}}\right)^{\mathrm{d} \ln T / \mathrm{d} \ln P}=10^{3.75} \mathrm{~K}$, already very close to the temperature of the irradiated layer of $10^{3.8} \mathrm{~K}$.

With the value for $v$ and $\Delta V_{\mathrm{s}}^{2}=0.1 V_{\mathrm{s}}^{2}=10^{10.6} \mathrm{~cm}^{2} / \mathrm{s}^{2}$ the drift velocity into the shadow zone becomes

$\left|v_{y}\right|=10^{2.9} \mathrm{~cm} / \mathrm{s}$.

This means that the time for matter to drift from irradiated parts into the region below $\mathrm{L}_{1}$ becomes

$t_{\text {drift }}=\frac{l}{\left|v_{y}\right|}=10^{6.5} \mathrm{~s}=37^{\mathrm{d}}$.

This is longer than the duration of the outburst. It is also much longer than the cooling time of three days of the column considered. The matter therefore has cooled to temperatures in the shadow zone long before it reaches the region below $\mathrm{L}_{1}$.

Thus the shadowing of the mass transfer region by the accretion disk rim and the strong Coriolis force prevent any effect of irradiation of the secondary star on the mass transfer. This conclusion agrees with the observational evidence that is discussed in the preceding sections.

We discussed this here for the case of WZ Sge in particular, but we expect the same effect to be true for other CV systems as well.

\section{Smak's (1996) criticism and a new picture of the TTI model}

Smak $(1996,2000)$ argued that "the TTI model faces a serious problem with the sequence of events it predicts: the superhumps should appear at an early phase of a superoutburst (certainly not later than its maximum), while observations show that this happens only one or two days after maximum". In the original TTI model, the last normal outburst triggers a tidal instability, leading to an eccentric disk which causes a major enhancement of the accretion rate. Accordingly, the superhumps should appear in the very early phase of a superoutburst. Therefor Smak (1996) suggested instead a hybrid model (called EMT model) by combining the tidal instability with the enhanced mass-transfer model. In Smak's picture, irradiation of the secondary star by the central white dwarf and the boundary layer during the superoutburst causes enhanced mass transfer which now can keep the disk in hot state for much longer time. There is then enough time for the disk to develop the eccentric structure, a necessary condition as claimed by Whitehurst \& King (1991).

In response to Smak's criticism, we here propose a refinement of the TTI model. Before doing so, let us first examine the observations more carefully. Bateson (1977) classified the superoutburst light curves of VW Hyi into types S1 to S8 but these are basically two types; S1-S5 with simple superoutburst light curves with one continuous rise and fall and S6-S8 with light curves of a precursor-main outburst type. Almost all observers note that the rising part of the superoutburst is indistinguishable from that of the normal outburst. As discussed by Marino \& Walker (1979), in the case of the S6-S8 types the precursor normal outburst was separated by a dip from the main part of the superoutburst while it is already merged with the main superoutburst in the case of S1-S5. That is, in the type S1-S5 superoutbursts, the time delay between the triggering normal outburst and the bulk of the superoutburst is too short to be observable (Marino \& Walker 1979; van der Woerd \& van Paradijs 1987).

There are several examples in which the superoutburst light curve in optical light looked more or less as one single continuation but it consisted of a precursor and a main outburst at shorter wavelengths. In general, precursor part and main part of the superoutburst are more clearly separated as we go to shorter wavelengths. This can be seen in the Voyager farultraviolet observations of the 1984 October-November superoutburst of VW Hyi (Pringle et al. 1987), and in EUV observations of the 1997 March superoutburst of OY Car by Mauche \& Raymond (2000) and the 1997 October superoutburst of SW UMa by Burleigh et al. (2001). When the precursor is well separated from the main superoutburst, the main outburst grows in amplitude with the growth of the superhump light. The long duration of the superoutburst can not be the 
cause of the eccentric disk because the superhumps grow together with the rise to the main outburst. As far as superoutbursts of the precursor-main outburst type are concerned, observations agree very well with the TTI model.

We now turn to the S1-S5 type superoutbursts in which light curves look like a single continuation and the superhumps appear only one or two days after maximum and therefore Smak's criticism seems relevant. To deal with it, we here propose a refinement of the original TTI model that explains why superhumps appear a few days after the superoutburst maximum in some SU UMa stars. We suggest that these are those outbursts in which the last normal outburst occurs in full readiness for the superoutburst and that the normal outburst and the superoutburst are completely merged as explained below.

We first discuss the outburst duration in dwarf novae. In particular, let us address why in the TTI model the disk in normal outbursts can not stay hot even when much mass has already been stored in the disk for the coming superoutburst. The essential point of the TTI model lies in the explanation of the shortness of the normal outburst. When the normal outburst occurs, the disk jumps from the cold state to the hot state. The surface density distribution then changes from $\Sigma \propto r$ in the cold state to $\Sigma \propto r^{-0.75}$ in the hot state. On the other hand, the critical surface density below which no hot state exists varies with the radius as $\Sigma_{\text {crit }} \propto r$. Thus the outer rim of a free accretion disk (by "free" we mean not yet bound by the tidal removal of angular momentum) will always peter out to surface densities below the critical density below which no hot state is possible. When the heating wave released in the outburst reaches this critical surface density a cooling wave is set up that moves inward and cuts off the (normal) outburst. In the TTI model, the normal outburst is an outburst in which the disk's outer edge does not yet reach the 3:1 resonance radius and thus we have a rather ineffective tidal removal of angular momentum from the disk, explaining its short duration.

On the other hand, if tidal removal of angular momentum is very effective, the expansion of the disk is stopped, i.e., the disk hits a tidal wall, and the matter is dammed up there. If there is enough mass in the disk, the surface density at the tidal wall is above the critical surface density and the disk stays in the hot state, and the long viscous depletion of mass from the disk ensues. This explains the long duration of the outburst. In the case of $U$ Gem stars the tidal wall is provided at the ordinary tidal truncation radius, in the case of SU UMa stars at the 3:1 resonance radius, and in the case of WZ Sge at the 2:1 resonance.

Let us now come back to the problem of S1-S5 type superoutbursts. In SU UMa stars with relatively long orbital periods, near two hours, having binary mass ratios around 0.2 such as VW Hyi, the tidal truncation radius lies just beyond the 3:1 resonance radius. If the final normal outburst occurs in full readiness, the disk will not only expand to the 3:1 resonance radius but also pass it. The disk will then reach the tidal truncation radius so that its expansion is stopped there (see, the upper right-most panel of Fig. 4). The viscous depletion of matter (i.e., the viscous plateau stage of outburst) ensues even when the eccentric tidal instability (i.e., superhumps) has not yet developed to observable amplitude. One or two days after the light maximum, the tidal instability and the superhumps
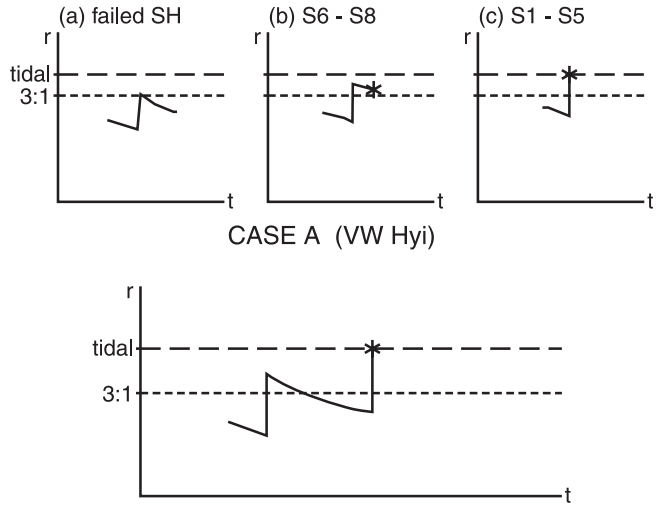

CASE B (most SU UMa)

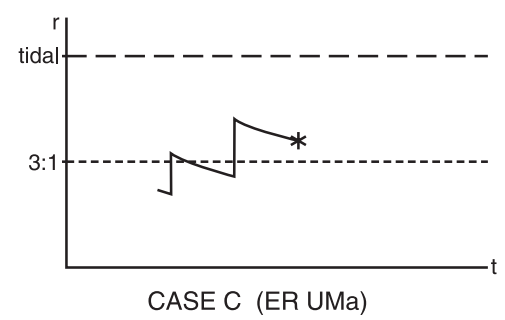

Fig. 4. Various ways in which superoutbursts are triggered (see text for details). Horizontal axis: time, vertical axis: radius. Short dashed line: 3:1 resonance radius, long-dashed line; tidal truncation radius, solid line: outer disk radius, asterisk: ignition of superoutburst. In order of decreasing mass ratio (increasing separation of tidal and 3:1 resonance radii, increasingly longer growth time of superhumps): case A: relatively large mass ratio around 0.2 (a), (b), and (c): ordinary outbursts occurring in various stage of readiness for a superoutburst in one and the same system. Case B: smaller mass ratio. Case C: extremely small mass ratio.

have grown to large amplitude, now taking over the role of tidal removal of angular momentum from the ordinary tidal torques at the truncation radius. This may be the reason why in these outbursts the superhumps grow one or two days after the maximum.

In the original suggestion of the TTI model (Osaki 1989, 1996), the importance of the tidal truncation radius just beyond the 3:1 resonance radius was not appreciated and only the precursor-main outburst type superoutburst was dealt with. With this new refinement we can now understand different aspects of superoutbursts of various SU UMa stars. Figure 4 illustrates how the last normal outbursts trigger the superoutburst under different conditions. Each panel schematically shows the time variation of the disk's outer edge. The asterisk denotes the point where the superoutburst is triggered. The uppermost three panels (case A) represent the longest orbitalperiod SU UMa stars with periods around two hours (typified by VW Hyi) and binary mass ratios $q$ around 0.2 for which the $3: 1$ radius and the tidal truncation radius are close to each other. The upper left panel illustrates an aborted superhump during the last normal outburst, which fails to develop to the superoutburst discussed in Sect. 2.2. The upper middle panel illustrates the case in which the last normal outburst first triggers a tidal instability and then the superoutburst develops together with the superhumps, corresponding to the precursor-main 
outburst type of S6-S8. The upper right panel illustrates the case of full-readiness in which the disk expands to reach the tidal truncation radius and the hot viscous plateau stage sets in. The tidal instability and the superhumps then develop to large amplitudes, corresponding to the S1-S5 case just discussed above.

The middle panel (case B) shows SU UMa stars with shorter orbital periods that have lower q values. In this case, even if the outer edge of the disk reaches the 3:1 resonance, the growth rate of the tidal instability, proportional to $q^{2}$, is so low that there is not enough time for the superhump to grow to large amplitude within the normal outburst duration. Once the normal outburst ends and the disk returns to quiescence, the outer edge of the disk contracts due to the addition of matter with low specific angular momentum and shrinks below the $3: 1$ resonance. Observationally, the normal outburst in this case is indistinguishable from those normal outbursts in which the disk's edge does not reach the 3:1 resonance at all. A next normal outburst will then push the disk edge to the tidal truncation radius. The hot viscous plateau stage sets in. The superhumps develop a few days after the light maximum. Most SU UMa stars correspond to this case B.

The bottom panel (case C) illustrates the case of the shortest orbital period SU UMa stars with the lowest mass ratio $q$ in which normal outburst occurs frequently. In this case the tidal truncation radius is far away from the 3:1 resonance radius. As discussed for case B, the normal outburst, in which the disk's outer edge reaches the 3:1 resonance, can not develop the superhumps to large amplitude. This may repeat in the next normal outburst as well. However, since the disk expands more and more above the 3:1 resonance radius, it may finally not shrink below the 3:1 resonance radius even during quiescence and the tidal instability continues to operate in quiescence. If sufficient time elapses, the tidal instability and the superhumps grow to large amplitude. Tidal heating at the outer edge of the disk will then force the upward thermal transition to the hot state, starting a new outburst. This outburst is of outside-in type and it develops always to a superoutburst because the disk is now fully eccentric, that is, a superoutburst triggered not by the normal outburst but rather by the tidal instability.

An obvious prediction in this case is that the superhump should be visible in large amplitude even during the rising branch of the outburst. This case (case C) corresponds to ER UMa stars with the shortest supercycle less than 50 days, and with the very short normal-outburst cycle, as short as 4 days. (The WZ Sge stars do not belong to this case because normal outbursts do not occur so frequently). It was a puzzle in observations of the ER UMa stars that superhumps in large amplitude were observed during the rising branch of the superoutburst (see observation of ER UMa by Kato et al. 1996 and V1159 Ori by Patterson et al. 1995). In the standard scenario of SU UMa stars, the superhumps were expected to appear later during the superoutburst the lower the mass ratio of the system is because of their increasingly longer growth time. The mystery of these paradoxical observations is now solved.

Although the 3:1 resonance and the ordinary tidal truncation both remove angular momentum from the disk via the secondary's tidal force, there exists an essential difference in nature between them. In a sense the 3:1 resonance is more active but the ordinary tidal truncation is more passive in nature: When the eccentric disk (and the superhump) is fully developed by the 3:1 resonance, tidal removal of angular momentum from the disk is very strong as demonstrated by Murray (1998) with SPH hydrodynamic simulation. This keeps the disk in the hot state longer. When the superoutburst ends, the quiescent disk becomes quite compact and far removed from the 3:1 resonance radius. On the other hand, the ordinary tidal truncation is passive in that it simply inhibits the disk to expand beyond the truncation radius but it does not cause the disk to contract far below that limit.

We conclude that the TTI model fully explains the sequence of events of SU UMa stars and that Smak's criticism is properly dealt with by the new refinement.

\section{The overall picture of the 2001 outburst of WZ Sge}

We now give a description of the overall development of the 2001 outburst of WZ Sge in terms of the TTI model, based on a very low viscosity in quiescence and no enhanced mass transfer during the outburst. In this latter point we differ from the observational account by Patterson et al. (2002). As shown in Sect. 2.3 their evidence for increased mass transfer is probably the result of a misinterpretation of orbital light curves in the common superhump era.

\section{(1) The main accretion event}

Osaki (1995) has already pointed out that the basic outburst energetics of WZ Sge fits to Osaki's (1974) disk instability model in its simplest form: long quiescence of about $30 \mathrm{yrs}$ coupled with a low mass transfer rate $\sim 10^{15} \mathrm{gs}^{-1}$ allows the disk to accumulate mass $\sim 10^{24} \mathrm{~g}$, which is dumped onto the central white dwarf during an outburst. Cannizzo (2001) has shown that the 2001 superoutburst of WZ Sge can be explained by viscous depletion of matter in a disk with an initial mass $\sim 10^{24} \mathrm{~g}$.

\section{(2) Quiescence and long recurrence time}

Two different models have been proposed to explain the long recurrence time of WZ Sge of about $30 \mathrm{yrs}$. One is a model with extremely low viscosity in quiescence (Smak 1993; Osaki 1995; Meyer-Hofmeister et al. 1998). The other is a model of a steady or almost steady cold disk with a standard value of viscosity in the cold state but with its inner part truncated (Warner et al. 1996; Hameury et al. 1997). Such a disk is unable to store the amount of mass required for the outburst. (See Fig. 3 of Meyer-Hofmeister et al. 1998 for a comparison of the two models.)

The missing mass in the second model would have to be supplied during the outburst. If $10^{24} \mathrm{~g}$ would be transferred on a time scale of one day the enhanced mass transfer rate should be $\dot{M}_{\text {tr }} \simeq 10^{19} \mathrm{gs}^{-1}$ and it may be rather unlikely to overlook such a large increase. As discussed in Sect. 2.3, there is no good observational evidence for enhanced mass transfer during the outburst. 
The very low quiescent viscosity in the first model can be understood by decay of magnetic turbulence (Gammie \& Menou 1998). In WZ Sge this occurs during the final decline to quiescence (Osaki et al. 2001). The extremely low viscosity value of WZ Sge stars may result as consequence of a cool brown dwarf secondary that cannot support a magnetosphere whose interaction with the accretion disk has been made responsible for the standard quiescent viscosity of ordinary dwarf novae (Meyer \& Meyer-Hofmeister 1999).

\section{(3) Start of outburst and early humps}

Least understood in this scenario is the initial kick to start the thermal instability in the case of extremely low quiescent viscosity. Once the thermal instability is started and the disk goes into the hot state, the standard high viscosity is most likely produced by the magneto- rotational instability (Balbus and Hawley instability).

A sudden jump to high viscosity makes the accumulated mass spread inwards and outwards and form a standard hot accretion disk. As discussed by Osaki \& Meyer (2002), the outer edge of the disk not only reaches the 3:1 resonance but passes it and reaches the 2:1 resonance radius where the two armed spiral pattern of the Lindblad resonance is excited. Increased tidal torques now stop the expansion of the disk and the tidal dissipation produces the observed photometric double humps (early humps, or "OOH” in Patterson et al. 2002).

\section{(4) Late appearance of common superhumps}

Within the first 12 days the large amount of mass residing near the 2:1 resonance region is gradually cleared by tidal loss of angular momentum. This enhances the relative importance of mass around the 3:1 resonance (responsible for the eccentric tidal instability). This now allows the disk to develop the common superhumps as discussed below.

Lubow (1991) has shown that an eccentric "corotation" resonance (different from the familiar eccentric Lindlad resonance) occurs at the 2:1 resonance through the tidal potential component $m=2$, which acts to damp eccentricity, in competition to the excitation of eccentricity at the 3:1 resonance. As long as a large amount of matter resides at the 2:1 resonance the damping effect will dominate, preventing the growth of eccentricity. As matter is cleared from the 2:1 resonance region, the excitation effect at the $3: 1$ resonance will increase in relative importance and eventually win. The disk eccentricity will grow to large amplitude and superhumps appear.

The tidal removal of angular momentum by the eccentric disk now takes over the role of the 2:1 resonance. The common superhump era corresponds to this stage. Thus the 2:1 resonance not only offers an explanation for the early hump phenomenon but it may also act as a suppressor of growth of the eccentricity in WZ Sge stars. The rather late appearance of the common superhumps in WZ Sge is then a natural consequence of the 2:1 resonance.

Light curves in the common superhump era were already discussed in subsection 2.3. Orbital humps and eclipses in this stage are due to the superhump light source itself and there is no evidence for enhanced mass transfer.

\section{(5) Late superhumps}

The superhump light signal often stays on after the end of the main superoutburst of SU UMa stars and the eccentric disk seems to survive for a while. Light variations with the superhump period after the end of the main superoutburst are called "late superhumps". The late-superhump phenomenon is conspicuous in WZ Sge stars, late superhumps continued to exist for one hundred days in the 2001 outburst of WZ Sge.

This phenomenon is most naturally explained by decoupling of the tidal instability from the thermal instability proposed by Hellier (2001). He suggested that in a binary system with extremely small mass ratio the tidal torques on the eccentric disk are not strong enough to keep the disk in the hot state though the tidal eccentric instability still goes on. The premature shutdown of the thermal instability ends the superoutburst even when the disk still has its eccentric form.

When near the transition into quiescence the superhump signal has dwindled away and the ordinary hot spot becomes dominant but the disk still retains a residual eccentricity a modulation in phase and intensity of the hot spot light occurs as the impact point of the accretion stream travels around the elliptically deformed circumference of the disk together with the revolving mass supplying secondary. This was documented by Rolfe et al. (2001) for the eclipsing dwarf nova IY UMa in the very late superhump phase.

\section{(6) Dip and Echo outbursts}

The WZ Sge stars often show rebrightening or reflare after the end of the main outburst, the most spectacular case was six consecutive rebrightenings of EG Cnc in 1996/1997. The 2001 outburst of WZ Sge showed the same phenomenon but it had as many as 12 short outbursts with a mean repetition time as short as 2 days. One of the important aspects during the echo outburst era is that the superhump light signal continues to exist throughout this period even to the final decline stage and that the superhump amplitude stayed approximately constant in intensity while the background light intensity was highly variable.

Osaki et al. (2001) and Hellier (2001) proposed that echo outbursts of EG Cnc are normal outbursts in the disk where tidal removal of angular momentum from the mass reservoir beyond the 3:1 resonance radius feeds mass into the inner disk and repeatedly excites the thermal instability. Note that no enhanced mass transfer is needed. In particular, Osaki et al. (2001) demonstrated that this model can explain the sudden cessation of echo outbursts as due to decay of magnetic viscosity in the disk. This model can be applied to the 2001 outburst of WZ Sge as well. The only difference being the amount of mass in the reservoir, larger in WZ Sge than in EG Cnc.

On the other hand, Hameury et al. (2000) have proposed enhanced mass transfer to explain echo outbursts. Patterson et al. (2002) supported this model because they saw observational evidence for enhanced mass transfer. However, as discussed in Sect. 2.3, their conclusion for enhanced mass transfer is simply due to a misinterpretation of their observations. There is no evidence for enhanced mass transfer and the supporting evidence disappears. 
Enhanced mass transfer also tends to quench an existing eccentric structure (see Ichikawa et al. 1993; Lubow 1994) since addition of a large amount of mass with low specific angular momentum leads to disk shrinkage. This would contradict the observed long endurance of late superhumps in WZ Sge and EG Cnc which may result just because of the low mass transfer rate in these systems compared to ordinary SU UMa stars.

\section{(7) Final decline}

As discussed by Osaki et al. (2001), the final decline is understood in terms of decay of magnetic turbulence in the cold disk. WZ Sge has entered into dormancy for another 20 or $30 \mathrm{yrs} \mathrm{ac-}$ cumulating mass in the disk for the next outburst.

\section{Conclusion}

We have carefully analyzed old and new claims of evidence for increased mass overflow in outbursts of SU UMa stars. We find that all this evidence is not well founded, inconclusive, and partially inconsistent with the observations themselves. Our theoretical analysis supports the conclusion of no increased mass overflow during the outbursts. In all cases we find that a refined thermal-tidal instability model with no increased mass overflow gives a consistent and convincing explanation of the observational results. This appears to rule out theoretical models for SU UMa star outbursts that postulate increased mass overflow during outbursts, and allows a deeper insight into the nature of these outbursts.

Acknowledgements. We would like to thank Emmi Meyer-Hofmeister for helpful discussions and technical assistance. Yoji Osaki acknowledges financial support from the Japanese Ministry of Education, Culture, Sports, Science and Technology with a Grant-in Aid for Scientific Research No. 12640237. We thank the referee for suggesting figures and solliciting comments on other SU UMa stars which significantly improved our presentation of the paper.

\section{Appendix A: Comments on Kato's (2002) model for early humps}

In the context of the general outburst model for WZ Sge we comment here on Kato's (2002) model for the early humps of the 2001 outburst of WZ Sge, an alternative model to ours. During the early stage of the 2001 outburst of WZ Sge, a twoarmed arch-like pattern was observed in the Doppler maps of emission lines of He II $4686 \AA$ and C III/N III on the second day of the outburst, July 24, (Baba et al. 2002) and on July 28 (Steeghs et al. 2001a). Kato (2002) has proposed that this and the early hump phenomenon can be understood in terms of irradiation of the disk by the central radiation source. In this picture, the irradiation occurs preferably at the elevated surface of the disk, which in turn is produced by vertical tidal deformation (Smak 2001; Ogilvie 2002). Thus the main energy source of early humps in Kato's model is the irradiation of a tidally elevated disk while it is the tidal dissipation in our model. The elevated disk is produced in his model by the vertical resonance discussed by Ogilvie (2002) while the 2:1 Lindblad resonance is responsible for the enhanced tidal dissipation in our model.
Here we comment on his interpretation. In our previous paper (Osaki \& Meyer 2002), we have only dealt with photometric humps of the 2001 outburst of WZ Sge and we have not discussed its Doppler maps. Here we present our view on the two-armed arch-like pattern in Doppler maps and its relation to the photometric humps. In our picture, the photometric humps are thought to be produced by the two-armed spiral pattern of the tidal dissipation due to the 2:1 Lindblad resonance. The enhanced tidal dissipation may then produce a vertical thickening of the accretion disk in a similar azimuthal two-armed pattern. Emission lines observed during the outburst of dwarf novae, in particular, those of He II at $4686 \AA$ and C III/N III complex at $4640 \AA$ observed on July 24 and on July 28 in the 2001 outburst of WZ Sge are most likely produced by the irradiation of the vertically elevated disk by hard photons (EUV and soft and hard X-rays) from the central source, in the high atmosphere of the accretion disk either by direct ionization and recombination or by chromospheric emission due to the temperature inversion as discussed by Smak (1991). We agree in this sense with Kato (2002) in that emission lines of He II $4686 \AA$ and $\mathrm{C} \mathrm{III/N} \mathrm{III} \mathrm{are} \mathrm{produced} \mathrm{by} \mathrm{irradiation} \mathrm{of} \mathrm{the} \mathrm{accretion} \mathrm{disk}$ by hard photons from the central radiation source. However, we do not agree with Kato (2002) on the explanation of the early hump phenomenon. Although two-armed arch-like pattern in the Doppler maps and the double-humped light curve (i.e., early humps) may be somehow related with each other, it is not so obvious that these two phenomena are produced by the one and the same cause as suggested by Kato (2002) because emission mechanisms are not necessarily the same for emission lines and for the continuum radiation. It seems premature to make a one to one correspondence between photometric humps and features in Doppler maps.

In fact, there is observational evidence that the light source for photometric humps is not due to the irradiation of the disk but rather it is due to the tidal dissipation. The observations of the very first day of the outburst of WZ Sge (July 23, 2001) tell us this. On the day of discovery of the 2001 outburst of WZ Sge, Ishioka et al. (2002) had found the growing humps during the rapid rise to maximum. On the same day, CCD spectroscopic observations were performed by K. Ayani at Bisei observatory, as shown in Fig. 1 of Baba et al. (2002). It showed Balmer lines in absorption but no emission lines were found around the wavelengths of He II and the C III/N III complex. This indicates that the irradiation of the accretion disk by hard photons from the central part of the disk or from the central white dwarf has not yet started on the first day. This is easily understood in dwarf nova outbursts. On the very first day, the outer part of the accretion disk of WZ Sge was already in the hot and high-viscosity state and the disk was expanding both inward and outward. The outer edge of the disk reached the 2:1 resonance radius, thus triggering the Lindblad resonance and creating the photometric humps. The inner edge of the hot disk had not yet reached the central white dwarf on the first day. That is a phenomenon well known as "UV delay" in the dwarf nova outburst. This demonstrates that the photometric humps already observed at the very first day do not require any irradiation of the disk at all. 
Furthermore, we argue that the irradiating radiation from the central source most likely did not penetrate deeply into the photosphere of the accretion disk as discussed in Sect. 3. In fact, higher Balmer lines $\mathrm{H} \gamma$ and $\mathrm{H} \delta$ and neutral helium lines, i.e., lines of the photospheric origin, were found in absorption even on July 24 and on July 28 when He II at $4686 \AA$ and $\mathrm{C}$ III/N III complex were in emission (Baba et al. 2001) while $\mathrm{H} \alpha$ and $\mathrm{H} \beta$ lines exhibited highly variable absorption cores together with emission wings. This means that the heating source for continuous radiation must be deeply seated within the accretion disk rather than coming from the outside (as in the case of irradiation). The continuum radiation is thus most likely due to the tidal dissipation, an argument favoring our tidal dissipation model against Kato's irradiation model for the early humps.

Let us now turn our attention to the problem of the radial range of the disk at which the tidal pattern is produced in Kato's model and in our model. Besides a localisation at the tidal cutoff radius, Kato (2002) considers Ogilvie's (2002) vertical resonance as an explanation of the two-armed arch-like pattern observed in Doppler maps. The vertical resonance radius, $R_{\text {vertical }}$, in the accretion disk is given by (see, e.g., Lubow 1981; Stehle \& Spruit 1999)

$R_{\text {vertical }} / a=(1-\sqrt{1+\gamma} / 2)^{2 / 3}(1+q)^{-1 / 3}$,

where $\gamma$ is the adiabatic exponent between pressure and density for the disk matter, $a$ is the binary separation, and $q=M_{2} / M_{1}$ is the ratio of secondary star to primary star mass. It is interesting to note that this expression has the same mass-ratio dependence as the 3:1 resonance for the eccentric tidal mode and the 2:1 resonance for the Lindblad resonance only differing in the numerical factor. We estimate $R_{\text {vertical }} / a=0.323(1+q)^{-1 / 3}$ for $\gamma=5 / 3$, and 0.441 for $\gamma=1$, while $R_{3: 1} / a=0.481(1+q)^{-1 / 3}$ for the 3:1 eccentric resonance and $R_{2: 1} / a=0.630(1+q)^{-1 / 3}$ for the 2:1 Lindblad resonance. Thus for a reasonable value of $\gamma$, the vertical resonance radius lies well inside the $3: 1$ resonance radius.

If the two-armed arch-like pattern observed in Doppler maps of the 2001 outburst of WZ ge were produced by the vertical resonance as suggested by Kato (2002) and if the disk in the 2001 outburst of WZ Sge were truncated at this radius, the disk would never reach the 3:1 resonance radius and the common superhump phenomenon would not be expected. This is another difficulty of Kato's model.

A further difference between Kato's and our model lies in the expected absolute value of the velocity for the two-armed arch-like pattern in the Doppler map. The peak separation of double emission lines in quiescence of WZ Sge is known to be around $700 \mathrm{~km} \mathrm{~s}^{-1}$ (e.g., Mason et al. 2001). From phase-folded spectra or Doppler maps of He II and $\mathrm{H} \alpha$ emission lines shown in Fig. 3 of Baba et al. (2002) we find that the velocity of the peak-intensity features during the early-hump era of the 2001 outburst of WZ Sge is around $500 \mathrm{~km} \mathrm{~s}^{-1}$. If this velocity represents the Keplerian circular velocity, the outer edge of the disk expanded by a factor of about two during the outburst. Since the orbital velocity of the secondary star of WZ Sge was found to be around $500 \mathrm{~km} \mathrm{~s}^{-1}$ (Steeghs et al. 2001b) as well, the observed velocity of around $500 \mathrm{~km} \mathrm{~s}^{-1}$ for the two-armed features in Doppler maps means that they are produced very near to the Roche lobe of the primary star, much favoring the 2:1 resonance interpretation over that of the vertical resonance.

We note that the observational accuracy does not allow to discuss a difference between the radii of the $2: 1$ resonance and the ordinary tidal cut-off, since for very small mass ratios these two radii can lie very close together.

\section{References}

Alexander, D. R., Johnson, H. R., \& Rypma, R. L. 1983, ApJ, 272, 773

Baba, H., Sadakane, K., Norimoto, Y., et al. 2002, PASJ, 54, L7

Bateson, F. M. 1977, New Zealand J. Sci., 20, 73

Burleigh, M. R., Pye, J. P., Poulton, S. W., et al. 2001, MNRAS, 325, 1458

Cannizzo, J. K. 2001, ApJ, 561, L175

Cheng, F. H., Sion, E. M., Szkody, P., et al. 1997, ApJ, 484, L149

Gammie, C. F., \& Menou, K. 1998, ApJL, 492, L75

Haefner, R., Schoembs, R., \& Vogt, N. 1979, A\&A, 77, 7

Hameury, J.-M., Lasota, J.-P., \& Hure, J.-M. 1997, MNRAS, 287, 937

Hameury, J.-M., Lasota, J.-P., \& Warner, B. 2000, A\&A, 353, 244

Hellier, C. 2001, PASP, 113, 78

Hirose, M., \& Osaki, Y. 1990, PASJ, 42, 135

Ichikawa, S., Hirose, M., \& Osaki, Y. 1993, PASJ, 45, 163

Ishioka, R., Uemura, M., Matsumoto, K., et al. 2002, A\&A, 381, L41

Kato, T., Nogami, D., \& Masuda, S. 1996, PASJ, 48, L5

Kato, T. 1997, PASJ, 49, 583

Kato, T. 2002, PASJ, 54, L11

Kippenhahn, R., \& Weigert, A. 1990, Stellar Structure and Evolution (Springer)

Kolb, U., \& Ritter, H. 1990, A\&A, 236, 385

Kuulkers, E., Knigge, C., Steeghs, D., et al. 2002, in The Physics of Cataclysmic Variables and Related Objects, ed. T. Gaensicke,

K. Beuermann, \& K. Reinsch, ASP Conf. Ser., 261, 443

Lasota, J.-P. 2001, New Astron. Rev., 45, 449

Lin, D. N. C., \& Papaloizou, J. 1979, MNRAS, 186, 799

Lubow, S. 1981, ApJ, 245, 274

Lubow, S. 1991, ApJ, 381, 259

Lubow, S. 1994, ApJ, 432, 224

Marino, B. F., \& Walker, W. S. G. 1979, in Changing Trends in Variable Star Research, ed. F. M. Bateson et al., IAU Colloq., 46, 29

Mason, E., Skidmore, W., Howell, S. B., et al. 2001, MNRAS, 318, 440

Mauche, C. W., \& Raymond, J. C. 2000, ApJ, 541, 924

Meyer, F., \& Meyer-Hofmeister, E. 1982, A\&A, 106, 34

Meyer, F., \& Meyer-Hofmeister, E. 1983, A\&A, 121, 299

Meyer, F., \& Meyer-Hofmeister, E. 1999, A\&A, 341, L23

Meyer-Hofmeister, E., Meyer, F., \& Liu, B. F. 1998, A\&A, 339, 507

Murray, J. R. 1998, MNRAS, 297, 323

Nauenberg, M. 1972, ApJ, 175, 417

O'Donoghue, D. 1990, MNRAS, 246, 29

O'Donoghue, D. 1992, in Vina Del Mar Workshop on Cataclysmic Variable Stars, ed. N. Vogt, ASP Conf. Ser., 29, 136

Ogilvie, G. I. 2002, MNRAS, 330, 937

Osaki, Y. 1974, PASJ, 26, 429

Osaki, Y. 1985, A\&A, 144, 369

Osaki, Y. 1989, PASJ, 41, 1005

Osaki, Y. 1995, PASJ, 47, 47

Osaki, Y. 1996, PASP, 108, 39 
Osaki, Y., Meyer, F., \& Meyer-Hofmeister, E. 2001, A\&A, 370, 488 Osaki, Y., \& Meyer, F. 2002, A\&A, 383, 574

Patterson, J., Jablonski, F., Koen, C., et al. 1995, PASP, 107, 1183

Patterson, J., McGraw, J. T., Coleman, et al. 1981, ApJ, 248, 1067

Patterson, J., Masi, G., Richmond, M., et al. 2002, PASP, 114, 721

Pedlovsky, J. 1982, Geophysical Fluid Dynamics (New York: Springer)

Pringle, J. E., Bateson, F. M., Hassall, B. J. M., et al. 1987, MNRAS, 225,73

Rolfe, D. J., Haswell, C. A., \& Patterson, J. 2001, MNRAS, 324, 529

Semeniuk, I. 1980, A\&AS, 39, 29

Smak, J. I. 1991, in Structure and Emission Properties of Accretion Disks, ed. C. Bertout, S. Collin-Souffrin, \& J. P. Lasota et al. (Paris: Éditions Frontières), 247

Smak, J. 1993, Acta Astron. 43, 101

Smak, J. 1996, in Cataclysmic Variables and Related Objects, ed. A. Evans, \& J. H. Wood (Dordrecht: Kluwer), 45
Smak, J. 2000, New Astron. Rev., 44, 171

Smak, J. I. 2001, Acta Astron., 51, 295

Spruit, H. 2002, Sol. Phys., accepted [astro-ph/0209146]

Steeghs, D., Marsh, T., Kuulkers, E., \& Skidmore, W. 2001a

Steeghs, D., Marsh, T., Knigge, C., et al. 2001b, ApJ, 562, L145

Stehle, R., \& Spruit, H. C. 1999, MNRAS, 304, 674

Suleimanov, V., Meyer, F., \& Meyer-Hofmeister, E. 1999, A\&A, 350, 63

van der Woerd, H., \& van Paradijs, J. 1987, MNRAS, 224, 271

Vogt, N. 1983, A\&A, 118, 95

Warner, B. 1995, Cataclysmic Variable Stars (Cambridge: Cambridge University Press)

Warner, B., \& O’Donoghue, D. 1988, MNRAS, 233, 705

Warner, B., Livio, M., \& Tout, C. A. 1996, MNRAS, 282, 735

Whitehurst, R. 1988, MNRAS, 232, 35

Whitehurst, R., \& King, A. 1991, MNRAS, 249, 25 